\title{
On the Scattering of Waves by a Disk
}

By Albert E. Heins, Ann Arbor, Mich., U.S.A, ${ }^{1}$ ) and Richard C. MacCamy, Pittsburgh, Pa., U.S.A.2)

\section{Introduction}

This paper is concerned with the scattering of sound waves by a circular disk of so-called 'soft' material. The appropriate boundary value problem is the determination of a function $U(r, \theta, z)$ satisfying the equation

$$
U_{r r}+\frac{1}{r} U_{r}+\frac{1}{\gamma^{2}} U_{\theta \theta}+U_{z z}+k^{2} U=0
$$

with the condition

$$
U(r, \theta, 0) \text { for } r<1 .
$$

In addition $U(r, \theta ; z)$ is to be made up of an incident field $U_{0}(r, \theta, z)$, which is prescribed, and a term $U^{s}(r, \theta, z)$ satisfying a radiation condition at infinity.

An explicit solution of this problem is possible by means of spheroidal harmonics $[1]^{3}$ ) but the resulting formulae are exceedingly complicated. An alternative method is to formulate the problem as an integral equation. Using GREEN's formula one can derive the so-called 'Helmholtz representation' for the solution namely,

$$
U(r, \theta, z)=U_{0}(r, \theta, z)+\frac{1}{4 \pi} \int_{0}^{1} \int_{0}^{2 \pi} f(\varrho, \phi) \frac{e^{i k R}}{R} \varrho d \phi d \varrho
$$

with $R^{2}=r^{2}+\varrho^{2}-2 r \cos (\theta-\phi)+z^{2}$ and where $f(\varrho, \phi)$ denotes the discontinuity of $U_{z}$ on the disk, $z=0, r<1$. From (1.2) and (1.3) one obtains the integral equation,

$$
0=U_{0}(r, \theta, 0)+\frac{1}{4 \pi} \int_{0}^{1} \int_{0}^{2 \pi} f(\varrho, \theta) \frac{e^{i k R}}{R} \varrho d \varrho d \theta, \quad z=0, r<1 .
$$

Unfortunately a solution of (1.4) is not known and one is led to the question of approximations.

1) The University of Michigan, Department of Mathematics

2) The Carnegie Institute of Technology, Department of Mathematics.

3) Numbers in brackets refer to References, page 264. 
When $k$ is set equal to zero the above problem becomes that of an electrified disk in electrostatics, simple solutions of which have long been known. It was observed by Copson [2] that in the case $k=0$ the equation (1.4) itself can be solved explicitly. Copson's procedure calls for the expansion of all quantities in a Fourier series of the angle $\theta$ and then carrying out the angular integrations in (1.4) to obtain simpler integral equations which can be solved. For the case of axially symmetric problems, that is, when $U$ does not depend on $\theta$ the method of Copson was extend by Jones [3]4) to equation (1.4) for arbitrary $k$. In this case an explicit solution is no longer possible but rather Jones was able to derive from (1.4) a regular Fredholm integral equation of second kind which is well adapted to an iteration scheme for small $k$.

It is rather an involved procedure to carry out the angular integration in (1.4) for $k \neq 0$. It proved necessary to use some rather complicated relations involving Bessel functions. Curiously enough, these complications vanished at the end and the resulting equation involved only elementary functions. The authors were led to believe that there should be also a simpler method for deriving this equation and this, in fact, proves to be true. The central feature of the problem appears to be the fact that an axially-symmetric solution of (1.1) is determined uniquely by its values on the axis of symmetry, $r=0$. Such a determination was observed by HENRICI [4] and further investigated by the authors in [5]. In this paper we show that an integral equation analogous to that of Jones may be derived without the intermediate step of rewriting the $\phi$ integration in (1.4). Further, the analysis is simple enough to be easily carried over to the case of non-symmetry about $r=0$.

In section 2 we indicate how the general situation without symmetry can be reduced to a series of problems similar to the axially symmetric case. In section 3 the necessary material from [4] is presented. In the next two sections we outline our method of solution and in the last section we carry out some simple calculations for the case in which $U_{0}(r, \theta, z)$ represents a plane wave of arbitrary angle of incidence. We include an approximate formula for the discontinuity of the $z$ derivative of $U$ on the disk, as a function of angle of incidence, when the wave number $k$ is small.

\section{Preliminary Remarks}

Solutions of (1.1) which are of class $C^{(2)}$ in a region are known to be analytic functions of $z$ and $x=r \cos \theta, y=r \sin \theta$. The particular function $U$ satisfying (1.2) will accordingly be analytic except on the ring $r=1, z=0$, so that the apparent singularity at $\boldsymbol{r}=0$ does not in reality appear. In this connection we shall have frequent use of the following lemma:

4) Jones studied the problem with the condition $\partial U / \partial z=0, z=0$ but the changes to the present problem are not difficult. 
Lemma 1. Let $G(x, y, z)$ be an analytic function for $x^{2}+y^{2}<R^{2}$. Then with $x=r \cos \theta, y=r \sin \theta$,

$$
G=\sum_{n=0}^{\infty}\left[A_{n}(r, z) \cos n \theta+B_{n}(r, z) \sin n \theta\right],
$$

where

$$
A_{n}(r, z)=r^{n} a_{n}(r, z), \quad B_{n}(r, z)=r^{n} b_{n}(r, z),
$$

$a_{n}$ and $b_{n}$ being continuous at $r=0$.

That $G$ can be expanded in a Fourier series is immediate. Further since $G$ is analytic,

$$
G=\sum_{j=0}^{\infty} \sum_{m=0}^{\infty} \sum_{n=0}^{\infty} a_{j m n} x^{j} y^{m} z^{n}=\sum_{j=0}^{\infty} \sum_{m=0}^{\infty} \sum_{n=0}^{\infty} a_{j m n} r^{j+m} \cos ^{j} \theta \sin ^{m} \theta z^{n} .
$$

Now the Fourier series for $G$ can be obtained by expanding $\cos ^{j} \theta$ and $\sin ^{m} \theta$ in multiple angles. However $\cos ^{j} \theta \sin ^{m} \theta$ will contribute to $\cos n \theta$ or $\sin n \theta$ only if $j+m \geqq n$. It follows that the coefficients of $\cos n \theta$ and $\sin n \theta$ involve only terms in $r^{n}$ or higher powers of $r$.

On the basis of lemma 1 we can write for the function $U$,

$$
\begin{aligned}
& U(r, \theta, z)=\sum_{n=0}^{\infty}\left[U^{n}(r, z) \cos n \theta+V^{n}(r, z) \sin n \theta\right] ; \quad U^{n}=r^{n} u^{n}, V^{n}=r^{n} v^{n}, \\
& U_{0}(r, \theta, z)=\sum_{n=0}^{\infty}\left[U_{0}^{n}(r, z) \cos n \theta+V_{0}^{n}(r, z) \sin n \theta\right] ; \quad U_{0}^{n}=r^{n} u_{0}^{n}, V_{0}^{n}=r^{n} v_{0}^{n}
\end{aligned}
$$

We write also,

$$
f(\varrho, \phi)=\sum_{n=0}^{\infty} c_{n}(\varrho) \cos n \phi+s_{n}(\varrho) \sin n \phi .
$$

Since the function $U$ is to be a solution of (1.1) the functions $U^{n}$ and $V^{n}$ are solutions of the equations,

$$
W_{r r}+\frac{1}{r} W_{r}+W_{z z}+\left(k^{2}-\frac{n^{2}}{r^{2}}\right) W=0
$$

from which it follows that the functions $u^{n}$ and $v^{n}$ are solutions of

$$
w_{r r}+\frac{2 n+1}{r} w_{r}+w_{z z}+k^{2} w=0 .
$$

If the series (2.1) and (2.2) are substituted in the Helmholtz formula (1.4) one finds easily that the terms corresponding to the various Fourier coefficients 
separate. In fact if we introduce the operators

$$
\begin{aligned}
& M_{n}(r, z ; g)=\frac{1}{4 \pi} \int_{0}^{1} \int_{0}^{2 \pi} g(\varrho) \varrho \cos n \phi \frac{e^{i k R}}{R} d \phi d \varrho, \\
& N_{n}(r, z ; g)=\frac{1}{4 \pi} \int_{0}^{1} \int_{0}^{2 \pi} g(\varrho) \varrho \sin n \phi \frac{e^{i k R}}{R} d \phi d \varrho,
\end{aligned}
$$

with $R^{2}=r^{2}+\varrho^{2}-2 r \varrho \cos \phi+z^{2}$, formula (1.3) becomes,

$$
U^{n}(r, z)=U_{0}^{n}(r, z)+M_{n}\left(r, z ; c_{n}\right), \quad V^{n}(r, z)=V_{0}^{n}(r, z)+M_{n}\left(r, z ; s_{n}\right) .
$$

We now proceed to derive one of the two key formulae in our work. We prove

$$
n ! u^{n}(0, z)=n ! u_{0}^{n}(0, z)+(-)^{n} \frac{\partial^{n}}{\partial\left(z^{2}\right)^{n}} \int_{0}^{1} c_{n}(\varrho) \frac{\varrho^{n+1} e^{i \mu^{\mu}}}{\mu} d \varrho, \quad n \neq 0,
$$

where $\mu^{2}=\varrho^{2}+z^{2}$. The same relation exists between $v^{n}, v_{0}^{n}$ and $s_{n}$. In order to verify $(I)$ we start with the observation that $U^{n}=r^{n} w^{n}$ implies,

$$
n ! u^{n}(0, z)=\left.\frac{\partial^{n} U^{n}}{\partial r^{n}}\right|_{r=0}, \quad n ! u_{0}^{n}(0, z)=\left.\frac{\partial U_{0}^{n}}{\partial r^{n}}\right|_{r=0} .
$$

Thus we are required to compute first the successive derivatives with respect to $r$ of $M_{n}\left(r, z ; c_{n}\right)$. In order to carry out these differentiations we write,

$$
\frac{e^{i k R}}{R}=F\left(\beta+z^{2}\right), \quad \beta=r^{2}+\varrho^{2}-2 r \varrho \cos \phi .
$$

We have then

$$
\beta_{r}=2 r-2 \varrho \cos \phi, \quad \beta_{r r}=2 \text { and } \quad \frac{\partial^{m} \beta}{\partial r^{m}}=0 \quad \text { for } m \geq 3 .
$$

Then we assert,

$$
\frac{\partial^{n}}{\partial r^{n}} \cdot \frac{e^{i k R}}{R}=\sum_{j=0}^{[n / 2]} c_{j}^{n} F^{(n-j)} \beta_{r}^{n-2 ;} \beta_{r r}^{i}, \quad c_{0}^{n}=1 .
$$

We prove (2.6) by the method of induction. It is clearly true for $\mathrm{n}=1$. Let us assume now that it is true for any $n>1$. Then

$$
\frac{\partial^{n+1}}{\partial \eta^{n+1}} \cdot \frac{e^{i k R}}{R}=\sum_{j=0}^{[n / 2]} c_{j}^{n} F^{n-j+1} \beta^{n-2 j+1} \beta_{r r}^{j}+\sum_{j=0}^{[n / 2]} c_{j}^{n} F^{n-j} \beta_{r}^{n-2 j-1} \beta_{r r}^{i+1}(n-2 j)
$$

since $\partial^{3} \beta / \partial r^{3}=0$. Each term in the second sum has the same form as a term 
in the first sum except the last one. This one is

$$
c_{[n] 2]}^{n} F^{n-[n / 2]} \beta_{r}^{n-2[n / 2]-1} \beta_{r y}^{[n / 2]+1}(n-2[n / 2]) .
$$

Now if $n$ is even, $[n / 2]=[(n+1) / 2]$ and $n-2[n / 2]=0$ so that (2.6) holds for $n+1$. If $n$ is odd, $n-2[n / 2]=1$ and $[(n+1) / 2]=[n / 2]+1$ so that the last term becomes

$$
c_{[n / 2]}^{n} F^{n+1-[(n+1) / 2]} \beta_{r}^{n+1-2[(n+1) / 2]} \beta_{r r}^{[(n+1) / 2]},
$$

which is the last term of (2.6) for $n+1$. We note that the coefficient of $c_{0}^{n}$ remains unchanged and hence always has the value unity.

Inserting (2.6) into (2.5), we have

$$
\begin{aligned}
\frac{\partial^{n} U^{n}(r, z)}{\partial \gamma^{n}}= & \frac{\partial^{n} U_{0}^{n}(r, z)}{\partial \gamma^{n}} \\
& +\frac{1}{4 \pi} \sum_{j=0}^{[n / 2]} c_{j}^{n} \int_{0}^{1} \int_{0}^{2 \pi}\left[c^{n}(\varrho) \varrho \cos n \phi F^{(n-j)}(2 r-2 \varrho \cos \phi)^{n-2 j} 2^{j} d \phi d \varrho\right]
\end{aligned}
$$

or

$$
\begin{aligned}
& n ! u^{n}(0, z)=n ! u_{0}^{n}(0, z) \\
& \quad+\frac{1}{4 \pi} \sum_{j=0}^{[n / 2]} c_{j}^{n}(-1)^{n} 2^{(n-j)} \int_{0}^{1} \int_{0}^{2 \pi} c^{n}(\varrho) \varrho^{n-2 j+1} \cos ^{n-2 j} \phi \cos n \phi F^{n-2 j} d \phi d \varrho .
\end{aligned}
$$

But

$$
\cos ^{\alpha} \phi=\frac{1}{2^{x-1}} \cos \alpha \phi+a_{1} \cos (\alpha-2) \phi+\cdots
$$

so that the only term which will survive integration with respect to $\phi$ is the term $j=0$. We have then

and

$$
n ! u^{n}(0, z)=n ! u_{0}^{n}(0, z)+\frac{(-1)^{n}}{4} \int_{0}^{1} c^{n}(\varrho) \varrho^{n+1} F^{n} d \varrho, \quad n \neq 0
$$

$$
U^{0}(0, z)=U_{0}^{0}(0, z)+\frac{1}{2} \int_{0}^{1} c^{0}(\varrho) \varrho F^{0} d \varrho .
$$

But $F$ is a function of $\beta+z^{2}$ and so the result $(I)$ follows.

We remark that the apparent difficulty in $(I)$ for $z=0$ is only illusory. For by lemma 1

$$
C^{n}(\varrho)=\varrho^{n} c^{n}(\varrho) \quad \text { so that } \quad c^{n}(\varrho) \varrho^{n+1} \frac{\partial^{n}}{\partial\left(z^{2}\right)^{n}} \cdot \frac{e^{i k \mu}}{\mu}
$$

remains bounded for $z=0$. This remark is important for our later results. 


\section{Axially Symmetric Solutions}

For $n=0$ and $k=0$ equation (2.4) becomes the equation for axially symmetric potential functions. It is well known that such functions are uniquely determined by their values on the axis of symmetry, provided those functions are analytic. In fact in terms of those functions the solution can be written down explicitly in the form,

$$
u(r, z)=\int_{-r}^{r} \frac{w(0, z+i \varrho)}{\sqrt{r^{2}-\varrho^{2}}} d \varrho .
$$

These facts can be extended to the general equation $(2.4)_{n}$, as discussed by HenRICI [4]. Again a solution is determined by its values on $r=0$, provided these functions are analytic. Further, the solution can again be written down in the form,

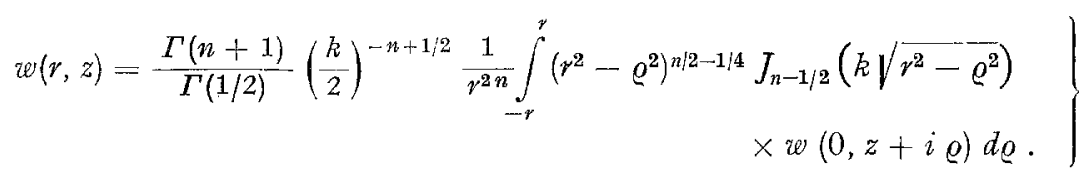

Equation (3.2) shows that actually a complete knowledge of $w(0, \varrho)$ is not necessary, but rather that only $w(0, z+i \varrho)+w(0, z-i \varrho)$ need be known.

We obtain our second fundamental relation by setting $z=0$ in (3.2), thus obtaining

$$
\left.\begin{array}{rl}
w(r, 0)=\frac{\Gamma(n+1)}{\Gamma(1 / 2)}\left(\frac{k}{2}\right)^{-n+1 / 2} \frac{1}{\gamma^{2 n}} \int_{0}^{r}\left(r^{2}\right. & \left.-\varrho^{2}\right)^{n / 2-1 / 4} J_{n-1 / 2}\left(k \sqrt{r^{2}-\varrho^{2}}\right) \\
& \times[w(0, i \varrho)+w(0,-i \varrho)] d \varrho .
\end{array}\right\}
$$

Formula(II) is aVolterra integral equation for the quantity $w(0, i \varrho)+w(0,-i \varrho)$. Its exact solution is not of concern to us as we shall encounter only the case in which the left hand side is zero, and then $w(0, i \varrho)+w(0,-i \varrho)$ will be identically zero.

\section{Solution for Small Wave Number}

In this section we shall derive integral equations of second kind analogous to those of JonEs [3]. These are particularly well suited to approximations for small $k$. We first derive the equations formally and then show that they do indeed yield solutions of the problems. We content ourselves with the determination of the cosine terms, that is, the functions $U^{n}(\gamma, z)$, the extension to sine terms being clear. 
We begin with the formula (I) and write $z^{2}=-\alpha$. Upon integrating (I) $n$ times with respect to $\alpha$, we obtain

$$
\left.\begin{array}{rl}
n ! \int_{0}^{\alpha} \cdots \int_{0}^{\tau_{1}}\left[U^{n}\right. & \left.(0, i \sqrt{\tau})-U_{0}^{n}(0, i \sqrt{\tau})\right] d \tau \cdots d \tau_{n-1} \\
& +\Lambda_{0}+\Lambda_{1} \alpha+\cdots \Lambda_{n-1} \alpha^{n-1}=\frac{(-)^{n}}{8} \int_{0}^{1} K_{n}(\beta) \frac{e^{i k \sqrt{\beta-\alpha}}}{\sqrt{\bar{\beta}-\alpha}} d \beta
\end{array}\right\}
$$

where the $\Lambda$ 's are constants and $K_{n}(\beta)=\varrho^{n} c^{n}(\varrho), \varrho^{2}=\beta$. This expression was derived under the assumption that $\alpha$ was real and negative. We observe, however, that all of the quantities which occur in (4.1) are analytic functions of the complex variable $\alpha$, as long as $\alpha$ is not real and positive. If we choose the branch of $\sqrt{\beta-\alpha}$ which is positive for $\arg \alpha=0, \alpha<\beta$, the integral on the right side of (4.1) is analytic in the complex $\alpha$-plane cut along the real axis from 0 to 1 . Thus $u^{n}(0, i \sqrt{\alpha})$ will suffer a discontinuity on approach to the real axis, $0<\alpha<1$ from above or below. Indeed from (4.1) we have for $\arg \alpha=0$,

$$
\left.\begin{array}{rl}
n ! \int_{0}^{\alpha} \ldots \int_{0}^{\tau_{1}}\left[u^{n}(0, i \sqrt{\tau})+u^{n}\left(0, i \sqrt{\tau^{-}}\right)-u_{0}^{n}(0, i \sqrt{\tau})\right. & \left.-u_{0}^{n}\left(0, i \sqrt{\tau^{-}}\right)\right] d \tau \cdots d \tau_{n-1} \\
& +2 \sum_{m=0}^{n-1} \Lambda_{m} \alpha^{m}=T^{n}\left(\alpha ; K_{n}\right\rangle .
\end{array}\right\}
$$

Here $u^{n}\left(0, i \sqrt{\tau^{n}}\right)$ denotes the value which $u^{n}(0, i \sqrt{\tau})$ assumes, when $\arg \tau=0$, after a clockwise circuit of the origin and $T^{n}(\alpha ; g)$ denotes the operator,

$$
\left.\begin{array}{rl}
T^{n}(\alpha ; g) & =\frac{(-1)^{n}}{4}\left\{i \int_{0}^{\alpha} g(\beta) \frac{\sinh k \sqrt{\alpha-\beta}}{\sqrt{\alpha-\beta}} d \beta+\int_{\alpha}^{1} g(\beta) \frac{e^{i k \sqrt{\beta-\alpha}}}{\sqrt{\overline{\beta-\alpha}}} d \beta\right\} \\
& =\frac{(-1)^{n}}{4}\left\{i \int_{0}^{1} g(\beta) \frac{\sinh k \sqrt{\alpha-\beta}}{\sqrt{\alpha-\beta}} d \beta+\int_{\alpha}^{1} g(\beta) \frac{\cos k \sqrt{\beta-\alpha}}{\sqrt{\beta-\alpha}} d \beta\right\} .
\end{array}\right\}
$$

Our next observation is based on formula (II). Since $U=0$ uо $z=0, r<1$ we have also $u^{n}(r, 0)=0$ on $r<1$. Applying (II) to the functions $u^{n}$ we have accordingly,

$$
u^{n}(0, i r)+w^{n}(0,-i r)=0, \quad 0<r<1,
$$

since these quantities will be solutions of a homogeneous Volterra equation, but this means that for $\arg \tau=0$

$$
u^{n}(0, i \sqrt{\tau})+u^{n}\left(0, i \sqrt{\tau^{-}}\right)=0,
$$

so that the first iterated integral on the left side of (4.2) is zero. The iterated 
integrals of $u_{0}^{n}$ are known functions, so that except for the values of the constants $A_{m}$ the left side of (4.2) may be considered as known. For simplicity we write,

$$
n ! \int_{0}^{\alpha} \cdots \int_{0}^{\tau_{1}} u_{0}^{n}(0, i \sqrt{\tau})+u_{0}^{n}\left(0, i \sqrt{\tau^{-}}\right) d \tau \cdots d \tau_{n-1}=H^{n}(\alpha) .
$$

Now let us denote the two integrals on the right side of (4.3) by $T_{1}^{n}(\alpha ; g)$ and $T_{2}^{n}(\alpha ; g)$ respectively so that $(4.2)$ becomes

$$
H^{n}(\alpha)+\sum_{m=0}^{n-1} A_{m} \alpha^{m}=i T_{1}^{n}\left(\alpha ; K_{n}\right)+T_{2}^{n}\left(\alpha ; K_{n}\right) .
$$

Essentially the equation (4.4) for $n=0$ was obtained by Jones [3]. He made the observation that if one finds the inverse of the operator $T_{2}^{n}\left(\alpha ; M_{n}\right)$ and applies it to (4.4) the product $\left(T_{2}^{n}\right)^{-1} T_{1}^{n}$ is an operator which is simple. It is not essential that one works out the exact details of this process but for completeness we outline the details. If we write $\beta=1-\sigma$ and $\alpha=1-\gamma$, equation (4.4) becomes

$$
\begin{aligned}
& H(1-\gamma)+\sum_{m=0}^{n-1} A_{m}(1-\gamma)^{m} \\
& \quad=\frac{(-1)^{n}}{2}\left\{i \int_{0}^{1} K_{n}(1-\sigma) \frac{\sinh k \sqrt{\sigma-\gamma}}{\sqrt{\sigma-\gamma}} d \sigma+\int_{0}^{\sigma} K_{n}(1-\sigma) \frac{\cos k \sqrt{\gamma-\sigma}}{\sqrt{\gamma-\sigma}} d \sigma\right\} .
\end{aligned}
$$

Now we recognize that the last integral may be inverted with the aid of the Laplace transform to give us

$$
\begin{array}{r}
\frac{(-1)^{n}}{2}\left\{K_{n}(1-\sigma)+-\frac{i}{2 \pi} \int_{0}^{1} \frac{K_{n}(1-\gamma)}{\sqrt{\sigma}}\left[\frac{\sinh k(\sqrt{\gamma}+\sqrt{\sigma})}{(\sqrt{\gamma}+\sqrt{\sigma})}+\frac{\sinh k(\sqrt{\gamma}-\sqrt{\sigma})}{(\sqrt{\gamma}-\sqrt{\sigma})}\right] d \gamma\right\} \\
=\frac{1}{\pi} \cdot \frac{d}{d \sigma} \int_{0}^{\sigma} \frac{\cosh k \sqrt{\sigma-\gamma}}{\sqrt{\sigma-\gamma}} d \gamma\left\{H^{n}(1-\gamma)+\sum_{m=0}^{n-1} A_{m}(1-\gamma)^{m}\right\} .
\end{array}
$$

Upon writing $\sigma=y^{2}, \gamma=x^{2}$, we obtain

$$
\left.\begin{array}{l}
y K_{n}\left(1-y^{2}\right)+\frac{i}{\pi} \int_{-1}^{+1} x K_{n}\left(1-x^{2}\right) \frac{\sinh k(x+y)}{(x+y)} d x \\
=(-1)^{n} \frac{2}{\pi} \cdot \frac{d}{d y} \int_{0}^{y} \frac{\cosh k \sqrt{y^{2}-x^{2}}}{\sqrt{y^{2}-x^{2}}}\left\{x H^{n}\left(1-x^{2}\right)+\sum_{m=0}^{n-1} \Lambda_{m}\left(1-x^{2}\right) x\right\} d x
\end{array}\right\}
$$


The essential fact to observe about (4.5) is that it is a Fredholm integral equation of second kind with a regular kernel which is small when $k$ is small. The method of successive approximations will certainly apply and yield the solution as a power series in $k$ for $k$ sufficiently small. We remark that in the actual mechanical process of computing the successive terms one need not make the transformation to (4.5). Since the operator $T_{1}$ involves

$$
\frac{\sinh k \sqrt{\alpha-\beta}}{\sqrt{\alpha-\beta}}
$$

it will produce terms of one higher degree in $k$ than $T_{2}$. Clearly an iteration process may be based directly on (4.4). That is, if we assume

$$
K_{n}=\sum_{m=0}^{\infty} K_{n m} k^{m}
$$

then it is easily seen that the $K_{n m}$ will be determined by recursion in the form,

$$
\int_{\alpha}^{1} \frac{K_{n m}(\beta)}{\sqrt{\beta-\alpha}} d \beta=F\left(K_{n, m-1} \cdots K_{n, 0}\right)
$$

or inverting this simple integral equation ${ }^{5}$ )

$$
K_{n m}(\alpha)=-\frac{1}{\pi} \cdot \frac{d}{d \alpha} \int_{0}^{\alpha} F\left(K_{n, m-1} \cdots K_{n, 0}\right) d \beta .
$$

In the developments so far there has been no apparent difference between the various values of $n$. The complication which arises is, of course, contained in the determination of the constants $A_{m}$. For this we use the remark made in connection with formula $(\mathrm{I})$ that the quantities $c^{n}(\varrho)$ must vanish like $\varrho^{n}$ when $\varrho \rightarrow 0$. Recalling that

$$
M_{n}(\beta)=\beta^{n / 2} c^{n}(\sqrt{\beta})
$$

this means we must have $K_{n}(\beta)$ vanishing as $\beta^{n}$ when $\beta \rightarrow 0$, that is,

$$
\frac{d^{n}}{d \beta^{m}} K_{n}(\beta)=0 \text { at } \beta=0, m=0,1, \ldots, n-1 .
$$

Thus we attack (4.4) as follows. Define functions $P^{n}(\alpha), Q^{n n}(\alpha)$ as solutions of

$$
H^{n}(\alpha)=T^{n}\left(\alpha ; P^{n}\right), \quad \alpha^{m}=T^{n}\left(\alpha ; Q^{n m}\right), \quad m=0,1, \ldots, n-1 .
$$

Then choose constants $A_{0}, A_{1}, \ldots, A_{n-1}$ so that,

$$
\frac{d^{m}}{d \alpha^{m}}\left[P^{n}(\alpha)+\sum_{m=0}^{n-1} \Lambda_{m} Q^{n m}(\alpha)\right]=0 \quad \text { at } \quad \alpha=0, \quad m=0,1, \ldots, n-1 .
$$

5) This is easily done with Laplace transforms. 
It would follow then that

$$
K_{n}(\alpha)=P^{n}(\alpha)+\sum_{m=0}^{n-1} \Lambda_{m} Q^{n m}(\alpha)
$$

would be a solution of (4.4) satisfying the condition (4.6).

The equations (4.8) constitute a system of linear equations for the $A_{m}$ 's which will have a solution unless the determinant should happen to be zero. If this were so then the homogeneous system corresponding to (4.8) would have a nontrivial solution $\Lambda_{0}^{0}, \ldots, \Lambda_{m}^{0}$. Following the general verification procedure which we present shortly, one would find that if

then

$$
c_{n}^{0}(\varrho)=\varrho^{-n} \sum_{m=0}^{n-1} A_{m}^{0} Q^{n m}\left(\varrho^{2}\right),
$$

$$
U(r, 0, z)=M_{n}\left(r, z ; c_{n}^{0}\right) \cos n \theta
$$

is a solution of equation (1.1), satisfying a radiation condition and vanishing on $z=0, r<1$. The uniqueness theorem for the problem of section (1) then states that $\tilde{U}(r, 0, z)=0$ hence $c_{n}^{0}(\varrho)$ is identically zero and finally all the constants $A_{m}^{0}$ must be zero. The contradiction shows that the determinant of system (4.8) cannot vanish.

We turn now to the verification of the formal solution which we have derived. First we observe that the equations (4.7) do indeed have solutions at least for sufficiently small $k$. Now consider the function,

$$
K_{n}(\alpha)=P^{n}(\alpha)+\sum_{n=0}^{n-1} A_{m} Q^{n m}(\alpha)
$$

with the constants $\Lambda_{m}$ chosen so as to satisfy equations (4.8). We put $c_{n}(\varrho)=\varrho^{-n} K_{n}\left(\varrho^{2}\right)$ and consider the function,

$$
U(r, \theta, z)=\left[U_{0}^{n}(r, z)+M_{n}\left(r, z ; c_{n}\right)\right] \cos n \theta .
$$

$U(r, \theta, z)$ will then be a solution of (1.1). Hence it will be an analytic function and lemma 1 applies to yield,

$$
U_{0}^{n}(r, z)+M_{n}\left(r, z ; c_{n}\right)=r^{n} u^{n}(r, z),
$$

$u^{n}(r, z)$ being a solution of equation (2.4). But now $u_{0}^{n}$, $u^{n}$, and $c_{n}$ will be connected by the formula (I). Thus we find corresponding to (4.2)

$$
\begin{aligned}
n ! u^{n}(0, i \sqrt{\alpha})+u^{n}\left(0, i \sqrt{\alpha^{-}}\right)=n ! u_{0}^{n}(0, i \sqrt{\alpha})+u_{0}^{n}\left(0, i \sqrt{\alpha^{-}}\right) & \\
& +\frac{\partial^{n}}{\partial \alpha^{n}} T^{n}\left(\alpha ; K_{n}\right) .
\end{aligned}
$$


Note that the $n$ derivatives of $T^{n}\left(\alpha ; K_{n}\right)$ may be formed even at $\alpha=0$ because we have constructed $K_{n}(\alpha)$ to vanish like $\alpha^{n}$ as $\alpha \rightarrow 0$. Now $P^{n}(\alpha)$ and $Q^{n m}(\alpha)$ are solutions of the integral equations (4.7) with $H^{n}(\alpha)$ representing the $n$-fold iterated integral of $-\left[u_{0}^{n}(0, i \sqrt{\alpha})+u_{0}^{n}\left(0, i \sqrt{\alpha^{-}}\right)\right]$. Thus differentiating (4.7) $n$-times and comparing with (4.9) we deduce,

$$
u^{n}(0, i \sqrt{\alpha})+u^{n}\left(0, i \sqrt{\alpha^{-}}\right)=0 .
$$

We have only to enter the result (4.10) in formula (II) to conclude that $u^{n}(r, 0)=0$ for $r<1$. Thus $U(r, \theta, z)$ is the (unique) $n$-th Fourier cosine coefficient of the solution to the problem of section 1 .

We have thus exhibited a method for calculating approximately any finite number of terms in the expansion of the function $U(r, \theta, z)$ of section 1 . The degree to which an actual solution is approximated will depend of course on the ease with which the incident field, $U_{0}(r, \theta, z)$, may be approximated by a finite number of terms of its Fourier series.

It would perhaps be well to insert here a few remarks concerning the small $k$ approximation. Let us suppose that the function $H^{n}(\alpha)$ has the form

Then we write

$$
H^{n}(\alpha)=\sum_{m=0}^{\infty} H_{m}^{n} k^{m}
$$

$$
H_{N}^{n}(\alpha)=\sum_{m=\mathbf{0}}^{N} H_{m}^{n} k^{m}
$$

What the iteration procedure for small $k$ yields then are functions $P_{N}^{n}(\alpha), Q_{N}^{n m}(\alpha)$ such that

$$
H_{N}^{n}-T^{n}\left(\alpha ; P_{N}^{n}\right)=0\left(k^{N+1}\right) ; \quad \alpha^{m}-T^{n}\left(\alpha ; Q_{N}^{n m}\right)=0\left(k^{N+1}\right) .
$$

We choose constants $A_{m i}^{N}$ so that equations (4.8) are satisfied for the approximating functions $P_{N}^{n}$ and $Q_{N}^{n m}$. Then set

and consider

$$
c_{n}^{N}(\varrho)=\varrho^{-n} P_{N}^{n}\left(\varrho^{2}\right)+\sum_{m=0}^{n} \Lambda_{m}^{N} Q_{N}^{m n}(\alpha)
$$

$$
U^{N}(r, \theta, z)=\left[U_{0}^{n}(r, z)+M_{n}\left(r, z ; c_{n}^{N}\right)\right] \cos n \theta .
$$

Again $U^{N}$ will be a solution of (1.1) so we can write,

$$
U_{0}^{n}(r, z)+M_{n}\left(r, z ; c_{n}^{N}\right)=r^{n} u_{N}^{n}(r, z) .
$$

Then following our previous procedure we deduce that

$$
u_{N}^{n}(0, i \sqrt{\alpha})+u_{N}^{n}\left(0, i \sqrt{\alpha^{-}}\right)=0\left(k^{N+1}\right)
$$


which again by formula (II) yields

$$
U^{N}(r, \theta, 0)=0\left(k^{N+1}\right) \text { for } r<1 .
$$

Thus solution of equation (4.7) to terms in $k^{N}$ yields the Fourier coefficients of the function $U(r, \theta, z)$ of section (1) to the same degree of approximation.

\section{Diffraction by a Hard Disk}

The problem considered by Jones [3] was the diffraction of a sound wave by a hard disk, that is, the condition (1.2) is now replaced by the vanishing of the $z$-derivative on the disk. In this section we indicate briefly how our methods need to be modified to handle that case. We shall maintain the same notation as in the previous sections, with $U(r, \theta, z)$ to satisfy

$$
U_{z}(r, \theta, 0)=0 \text { for } \quad r<1 \text {, }
$$

instead of (1.2). Replacing (1.3) we have

$$
U(r, \theta, z)=U_{0}(r, \theta, z)+\frac{1}{4 \pi} \cdot \frac{\partial}{\partial z} \int_{0}^{1} \int_{0}^{2 \pi} f(\varrho, \phi) \frac{e^{i k R}}{R} \varrho d \phi d \varrho,
$$

where $f(\varrho, \phi)$ this time represents the discontinuity of $U$ itself on the disk. If we require that the solution be continuous it follows that

$$
f(\varrho, \phi) \rightarrow 0 \text { as } \varrho \rightarrow 1 .
$$

The functions $U, U_{0}$ and $f$ can be decomposed into Fourier coefficients as we have done in section 3 . In place of (5.2) we get by an integration over $z$

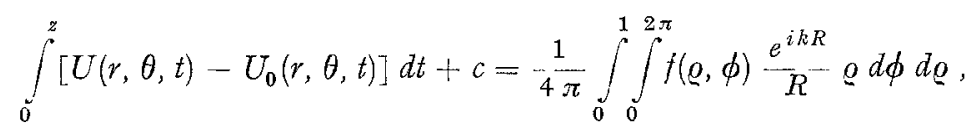

where $c$ is an arbitrary constant. The results of section 3 now carry over with minor modification. For example, when $r=0$

$$
\int_{0}^{z}\left[U(0, \theta, t)-U_{0}(0, \theta, t)\right] d t+c=\frac{1}{4 \pi} \int_{0}^{1} \int_{0}^{2 \pi} f(\varrho, \phi) \frac{e^{i k_{\mu}}}{\mu} \varrho d \phi d \varrho,
$$

where $\mu^{2}=\varrho^{2}+z^{2}$. If we write

$$
\int_{0}^{2 \pi} U(0, \theta, t) d \theta=u^{0}(t), \quad \int_{0}^{2 \pi} U_{0}(0, \theta, t) d \theta=u_{0}^{0}(t) \text { and } \int_{0}^{2 \pi} f(\varrho, \phi) d \phi=c_{0} .
$$


This last equation becomes

$$
\int_{0}^{3}\left[u^{0}(t)-u_{0}^{0}(t)\right] d t+c=\frac{1}{2} \int_{0}^{1} c_{0}(\varrho) \frac{e^{i k \mu}}{\mu} d \varrho .
$$

We now continue equation (5.4) into the domain of complex variables and we find that if $z \rightarrow i y(0<y<1)$ we get

$\int_{0}^{i y}\left(u^{0}(t)-u_{0}^{0}(t)\right] d t+c=\frac{1}{2}\left[\int_{0}^{y} c_{0}(\varrho) \varrho \frac{e^{-k \sqrt{y^{2}-\varrho^{2}}}}{i \sqrt{y^{2}-\varrho^{2}}} d \varrho+\int_{y}^{1} c_{0}(\varrho) \frac{e^{i k \sqrt{\varrho^{2}-y^{2}}}}{\sqrt{\varrho^{2}-y^{2}}} \varrho d \varrho\right]$, while if $z \rightarrow-i y(0<y<1)$ we get

$\int_{0}^{-i y}\left[u^{0}(t)-u_{0}^{0}(t) d t\right]+c=\frac{1}{2}\left[\int_{0}^{y}-c_{0}(\varrho) \frac{\varrho e^{k \sqrt{y^{2}-\varrho^{2}}}}{i \sqrt{y^{2}}-\varrho^{2}} d \varrho+\int_{y}^{1} c^{0}(\varrho) \frac{e^{i k \sqrt{\varrho^{2}-y^{2}}}}{\sqrt{\varrho^{2}-y^{2}}} \varrho d \varrho\right]$.

Upon adding these two equations and making some trivial changes of variables, we get

$$
\begin{aligned}
& \int_{0}^{y}\left[u^{0}(i t)-u^{0}(-i t)-u_{0}^{0}(i t)+u_{0}^{0}(-i t)\right] i d t+2 c \\
& =\left[\int_{0}^{1} \frac{i \sinh k \sqrt{y^{2}-\varrho^{2}}}{\sqrt{y^{2}}-\varrho^{2}} \varrho c_{0}(\varrho) d \varrho+\int_{y}^{1} \frac{\cos k \sqrt{\varrho^{2}-y^{2}}}{\sqrt{\varrho^{2}-y^{2}}} c_{0}(\varrho) \varrho d \varrho\right] .
\end{aligned}
$$

Now the boundary condition (5.1) implies, according to the representation (3.2), that $u^{0}(i t)-u^{0}(-i t)$ is a constant $c_{1}$. Hence equation (5.4) assumes the form

$$
\begin{aligned}
c_{1} y+2 c+\int_{0}^{y}\left[u_{0}^{0}(-i t)-u_{0}^{0}(i t)\right] i d l \\
\quad=\left[\int_{0}^{1} \frac{i \sinh k \sqrt{y^{2}}-e^{2}}{\sqrt{y^{2}-\varrho^{2}}} \varrho c_{0}(\varrho) d \varrho+\int_{y}^{1} \frac{\cos k \sqrt{\varrho^{2}-y^{2}}}{\sqrt{\varrho^{2}-y^{2}}} \varrho c_{0}(\varrho) d \varrho\right] .
\end{aligned}
$$

The constants $c$ and $c_{1}$ are evaluated from the requirements that $c_{0}(\varrho) \rightarrow 0$, $\varrho \rightarrow 1$ and $c_{0}(\varrho)$ is finite at $\varrho=0$. The equations involving the higher angular harmonics may be derived in much the same manner and indeed involve only further integration with respect to the variable $z^{2}=\left(-y^{2}\right)$ but we shall not pursue this matter further.

\section{Incident Plane Wave}

We include here some numerical results for the case of an incident plane wave for Dirichlet boundary conditions on the disk. There is clearly no loss 
of generality in assuming that the plane wave is incident upon the disk from the side $z<0$ and that its propagation normal lies in the $x-z$ plane. Let $\gamma$ denote the angle which this normal makes with the positive $z$ axis. The incident field in rectangular coordinates is then

$$
e^{i k(x \sin \gamma+z \cos y)}
$$

and in cylindrical coordinates it has the form $e^{i k(r \sin \gamma \cos \theta+z \cos \gamma)}$. Hence

$$
U(r, \theta, z)=e^{i \hbar(r \sin \gamma \cos \theta+z \cos \gamma)}+\frac{1}{4 \pi} \int_{0}^{1} \int_{0}^{2 \pi} f\left(\varrho, \theta^{\prime}\right) \frac{e^{i \hbar R}}{R} \varrho d \theta^{\prime} d \varrho .
$$

On the axis $r=0$, this representation assumes the form

$$
U(0, \theta, z)=e^{i k z \cos \gamma}+\frac{1}{4 \pi} \int_{0}^{1} \int_{0}^{2 \pi} f\left(\varrho, \theta^{\prime}\right) \frac{e^{i k \mu}}{\mu} \varrho d \theta^{\prime} d \varrho .
$$

Or, upon integrating over $\theta$ and $\theta^{\prime}$, we get

$$
u_{0}^{0}(0, z)=e^{i k z \cos \gamma}+\frac{1}{2} \int_{0}^{1} c^{0}(\varrho) \frac{e^{i k_{\mu}}}{\mu} \varrho d \varrho .
$$

If we continue $z$ into the domain of complex variables and use the fact that

$$
u^{0}(0, i \gamma)+u^{0}(-i \gamma)=0, \quad 0<\gamma<1,
$$

we get

$$
\left.\begin{array}{rl}
\cosh (k y \cos \gamma)+\frac{1}{2} \int_{y}^{1} \frac{\cos k \sqrt{t^{2}-y^{2}}}{\sqrt{t^{2}-y^{2}}} t c^{0}(t) d t \\
\quad+\frac{i}{2} \int_{0}^{1} \frac{\sinh k \sqrt{y^{2}-t^{2}}}{\sqrt{y^{2}-t^{2}}} t c^{0}(t) d t=0 .
\end{array}\right\}
$$

Upon rewriting equation (6.1) in the form which we described in section 4 , we obtain

$$
\left.\begin{array}{rl}
G^{\mathbf{0}}(\sigma)+\frac{i}{\pi} \int_{0}^{1} G^{0}(\beta)\left[\frac{\sinh k(\beta+\sigma)}{\beta+\sigma}+\frac{\sinh k(\beta-\sigma)}{\beta-\sigma}\right] d \beta \\
=-\frac{4}{\pi} \cdot \frac{d}{d \sigma} \int_{0}^{\sigma} \varrho d \varrho \cos \Psi\left(k \sqrt{1-\varrho^{2}} \cos \gamma\right) \frac{\cosh \left(k \sqrt{\sigma^{2}-\varrho^{2}}\right)}{\sqrt{\sigma^{2}-\varrho^{2}}} .
\end{array}\right\}
$$

Here $G^{0}(\sigma)=\sqrt{1-y^{2}} c^{0}(y)$ and $\sigma=\sqrt{1-y^{2}}$. Upon applying the Neumann 
iteration procedure we find for small $k$

$$
\begin{gathered}
c^{0}(\varrho)=-\frac{4}{\pi \sqrt{1-\varrho^{2}}}\left\{1-\frac{2 i k}{\pi}+k^{2}\left[\frac{\sin ^{2} \gamma}{2}+\varrho^{2} \cos ^{2} \gamma-\frac{\varrho^{2}}{2}-\frac{4}{\pi^{2}}\right]\right. \\
-i k^{3}\left[\frac{\cos ^{2} \gamma}{3 \pi}+\frac{7}{9 \pi}-\frac{\varrho^{2}}{3 \pi}-\frac{8}{\pi^{3}}\right] \\
+k^{4}\left[\frac{\cos ^{4} \gamma}{72}\left(8 \varrho^{4}-4 \varrho^{2}-1\right)+\frac{\cos ^{2} \gamma}{12}\left(1+\varrho^{2}-2 \varrho^{4}\right)\right. \\
+\frac{1}{24}\left(1-2 \varrho^{2}+\varrho^{4}\right)-\frac{1}{3 \pi^{2}}\left(6-2 \varrho^{2}+2 \cos ^{2} \gamma\right) \\
\left.\left.+\frac{16}{\pi^{4}}\right]\right\}+O\left(k^{5}\right) .
\end{gathered}
$$

In the limit $k_{z}=k$ (normal incidence) this result coincides with the one which BouWkamp [1] obtained.

For the first harmonic, we obtain the equation

$$
\left.\begin{array}{l}
-4 i k \sin \gamma\left[y \frac{\sinh k_{z} y}{k_{z}}-\frac{\cosh k_{z} y}{k_{z}^{2}}\right]+\Lambda_{1}^{1} \\
=\int_{y}^{1} \frac{\cos k \sqrt{t^{2}-y^{2}}}{\sqrt{t^{2}-y^{2}}} t^{2} c^{1}(t) d t+i \int_{0}^{1} \frac{\sinh k \sqrt{y^{2}-t^{2}}}{\sqrt{y^{2}-t^{2}}} t^{2} c^{1}(t) d t
\end{array}\right\}
$$

while for the second harmonic, we get

$$
\left.\begin{array}{l}
\int_{y}^{1} \frac{\cos k \sqrt{t^{2}-y^{2}}}{\sqrt{t^{2}-y^{2}}} t^{2} c^{2}(t) d t+i \int_{0}^{1} \frac{\sinh k \sqrt{y^{2}-t^{2}}}{\sqrt{y^{2}-t^{2}}} t^{2} c^{2}(t) d t \\
\quad=4 k_{z}^{2}\left[\frac{y^{2} \cosh k_{z} y}{k_{z}^{2}}-\frac{3 y \sinh k_{z} y}{k_{z}^{3}}+\frac{3 \cosh k_{z} y}{k_{z}^{4}}\right]+\Lambda_{1}^{2} \Lambda_{2}^{2} y^{2} .
\end{array}\right\}
$$

Here the $A$ 's are constants of integration. We observe that for all three cases which we consider here, that is, the zeroth, the first and the second harmonics, we are always solving the same type of integral equation, the only change being in the form of the non-homogeneous term. Equations (6.2) and (6.3) are solved in the same manner which we solved (6.2) save for the fact that we have to evaluate the constants of integration. These are evaluated by the requirement that $c^{1}(t)$ and $c^{2}(t)$ are finite at $t=0$. We omit the details and merely give the results. We have

$$
\begin{aligned}
& c^{1}(\varrho)=-\frac{8 i k \varrho \sin \gamma}{\pi \sqrt{1-\varrho^{2}}}\left\{1+\frac{k^{2}}{6}\left[\left(2 \varrho^{2}-1\right) \cos ^{2} \gamma-\left(1+\varrho^{2}\right)\right]\right\}+0\left(k^{4}\right), \\
& c^{2}(\varrho)=\frac{8 k^{2} \varrho^{2} \sin ^{2} \gamma}{3 \pi \sqrt{1}-\varrho^{2}}+0\left(k^{3}\right) .
\end{aligned}
$$


The terms are calculated to different orders of $k$ so that it may be possible to calculate the scattering cross section to terms correct to the order $k^{4}$ if need be.

The work on this paper was sponsored by the Office of Ordnance Research, US Army and the US Air Force Office of Scientific Research.

\section{REFERENCES}

[1] Bouwramp, C. J., Diffraction Theory, Rep. Progr. Physics, 17, 35-100 (1954).

[2] Copson, E. T., On the Problem of the Electrified Disk, Proc. Edinburgh math. Soc. 3, 14-19 (1947).

[3] Jones, D. S., A New Method for Calculating Scattering, with Particular Reference to the Circular Dish, Comm. pure and appl. Math. 9, 713-746 (1956),

[4] Henrici, P., Zur Funtionentheorie der Wellengleichung, Comm. math. Helv. 27, 235-296 (1953).

[5] Heins, A. E., and MacCamy, R. C., Axially Symmetric Solutions of Elliptic Differential Equations, Tech. Rep. No. 24, AFOSR Contract No. AF 49(638)-227 (Carnegie Institute of Technology).

\section{Zusammenfassung}

Es wird gezeigt, dass das Problem, die Beugung einer ebenen Welle an einer kreisförmigen Öffnung oder Scheibe zu bestimmen, auf die Lösung von regulären Fredholmschen Integralgleichungen zweiter Art zurückgeführt werden kann. Die Lösungen dieser Integralgleichungen liefern uns für die Scheibe im Falle der Neumannschen Bedingung die radiale Variation der Unstetigkeiten der Wellenfunktion und im Dirichletschen Falle die Unstetigkeiten ihrer normalen Ableitung. Ist das Produkt von Öfnungsradius und Wellenzahl klein, so können die Integralgleichungen gelöst werden. Für die Ableitung der Integralgleichungen verwenden wir einerseits die Poissonsche Darstellung für die Wellenfunktion und andererseits die Fortsetzung der Helmholtzschen Darstellung in die komplexe Ebene.

(Received: July 25, 1959.) 